\title{
Integrated Information System based on Industrial Standard Protocols
}

\author{
P. Marino, F. Poza, and J. Nogueira \\ Electronic Technology Department, \\ Applied Electronic Institute \\ University of Vigo \\ Vigo, Spain \\ Fax: +34-(9)86-46954 7, Tel: +34-(9)86-812 162 \\ e-mail:pmarino@uvigo.es
}

\begin{abstract}
The authors present a communications server implementation called CORE, for a communications equipment company, whose products are mounted and tested in a manufacturing plant controlled by a Token-Ring LAN (Marino and Poza, 1995). This server is being designed for connecting until 16 robots and 30 PLC's, in a first release, as a "black box" transparent to flexible manufacturing plants, allowing an integrated information system without device dependence.

Transparency is achieved, using CORE in a integrated information system through the above mentioned LAN, with industrial standard communication interfaces as EIA-RS232C and PROFIBUS (Field Bus). Serial interfaces are provided by multiport real-time cards as IBM's ARTIC (Marino, Poza and Nogueira, Jan-Feb. 1995), for connecting SCARA type robots. Field bus interfaces are provided by means of PLC's with PROFIBUS ports, for the SINEC L2 network from SIEMENS. Hardware structure of CORE is based in a $486 \mathrm{PC}$ with two multiport ARTIC real-time cards for 8 robots each (Marino, Poza and Nogueira, June 1995), one interface card for SINEC L2 field bus, and another card for Token-Ring (Marino, 1995) LAN (Figure 1). This set of COprocessor and REal-time cards made the name CORE to the server.
\end{abstract}

\section{Keywords}

LANs, client/server, field bus, standard interfaces, flexible manufacturing, multiport cards. 


\section{INTRODUCTION}

Hardware and software products from information technologies available for manufacturing SMEs, have evolved behind similar products for business SMEs. A handful of new paradigms such as, re-engineering, tele-economy, COLD (Computer Output to Laser Disk) technology, client/server model, virtual networks, object oriented languages, and so on, are pushing for a new conceptual model of an industrial SME, in order to benefit the whole company, faced a competitive market, by means of a suitable use of information technologies.

A sustained flow of information through all SME's sections with appropriate format, at a given time, in the right location, at least cost for retrieval, processing, transmission, and storage (COLD), is under the concept of integrated information systems. Re-engineering SME's process affects to understanding of their information system architectures, because traditional solutions from business SME's were based in management mainframes, whereas client/server solutions are based in networks (LANs) of distributed computers. Dramatic reductions in costs of network equipment and communications services (tele-economy) have a great potential for large increments in industrial SME's in terms of productivity, product quality, and reduction in manufacturing costs.

Therefore information system architectures based in LANs with client/server applications, seems to be the more cost effective and flexible solutions for integrated communications in manufacturing SMEs, where different production resources must be rearranged within short cycles (CNC machines, manipulators, robots, cells, etc.), new tasks must be quickly added (measurement, quality control, control of alarms, environment sensors, preventive maintenance, etc.), or management parameters must be included for analysing throughputs (personnel, energy, equipment, investments, stocks, etc.) in real-time.

Server technologies based in a PC as a platform with standard communications cards for different protocols, where each card is provided with APIs (Application Programming Interface) for existing operations systems, represents an economic and flexible implementation of client/server architectures for integrated information system in industrial SME's. A such system implementation is presented in this paper.

\section{SYSTEM FEATURES}

The elements of integrated information system for control, monitorization and supervision of manufacturing plant (Marino and Poza, 1995) are depicted in figure 1. There are two kind of devices in cells included in manufacturing plant: 7576 Scara robots from IBM, and SIMATIC S5-95U PLC's from SIEMENS. Each robot has a standard EIA-RS232C (Marino, 1995) serial asynchronous point-to-point connection, and the PLC's have a standard EIA-RS485 (Marino, 1995) with a hardware and software interface, that meets levels 1 and 2 from a german standard field bus (PROFIBUS, DIN 19245 part 1).

Two main type of communications with cells are possible through CORE: transaction and event. The first one is composed by a command to the cells and a reply from them. These transactions will always be started by a LAN node, and will be used for real-time control and 
COMMUNICATIONS SERVER (CORE)

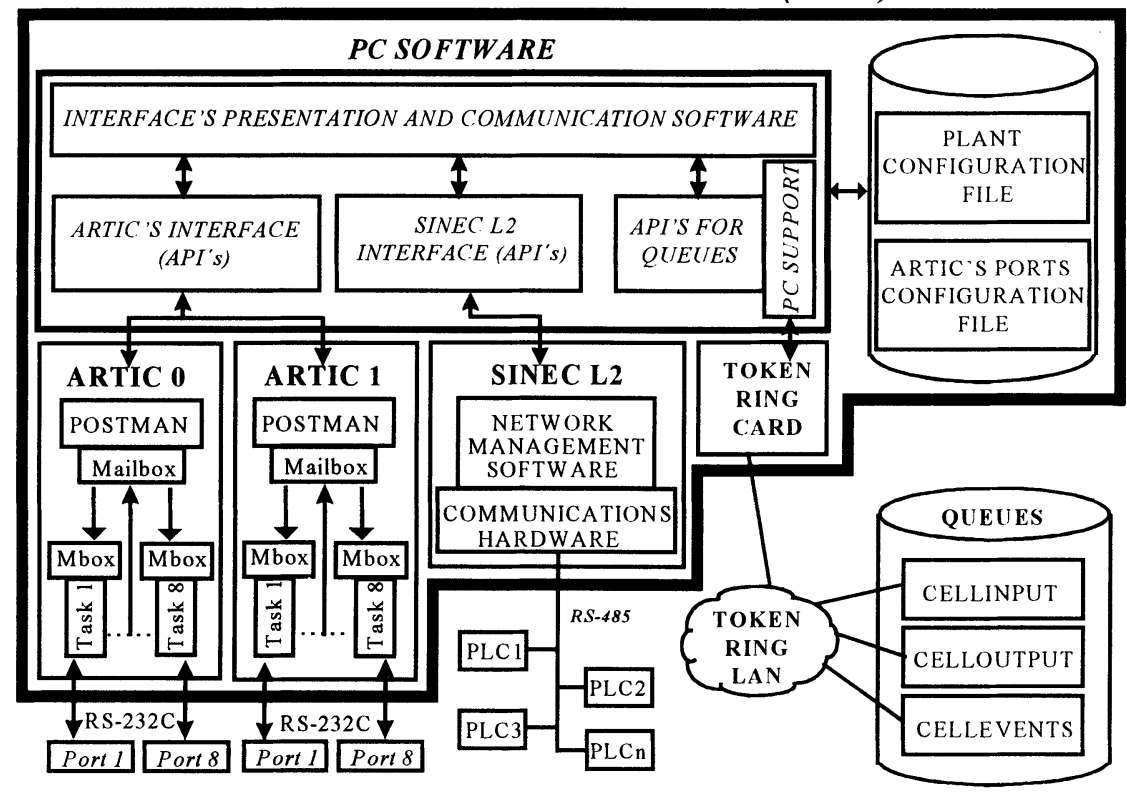

Figure 1 Hardware and software structure of CORE.

monitorization of manufacturing cells. Several commands have been defined (start, stop, load program, read data, write data, etc.) in abstract shape independent of specific devices.

The second type of communication (event) is started by manufacturing cells, and its origin is an event happened in their working (start of a product, end of a product, start of an alarm, end of an alarm, etc.), or a previous transaction (start, stop, etc.). Events include kinds of information like: event code, event time, manufacturing command, worker code, number of finished products, etc. This information allows a tracking of manufacturing plant performance.

All these communications will be made through a personal computer (PC) called communications server (CORE). Its main goals will be the communications routing between manufacturing cells and LAN of integrated information system, and the translation of defined abstract commands to suitable format for each specific type of device. For having these performances the PC includes (Figure 1): two ARTIC IBM cards with 8 RS-232C port each (Marino, Poza and Nogueira, Jan-Feb. 1995), a SIEMENS SINEC L2 card (CP 5412) and a Token Ring card (Marino, 1995).

The communication between LAN nodes and communications server (CORE) has like mediator the plant level company's AS/400 computer, for integration of information attending managerial requests. For this reason three data queues are defined in AS/400 with a specific format: cellinput, celloutput and cellevents. The first two are involved with transactions and the last one with events. Any LAN node can send data to manufacturing cells placing a command in the cellinput queue. CORE's software takes the command from the cellinput 
queue and send it to a manufacturing cell through its interface card (ARTIC 0, ARTIC 1 or SINEC L2). After the cell processing of received command, a reply is sent to CORE through its interface card and that put it in the celloutput queue. LAN node that created the transaction reads the reply from this queue. Events from any manufacturing cells are gathered for CORE and sent to cellevents queue. A program in the plant level company's AS/400 computer, takes the events from this queue and store them in one or several databases devoted to monitorization and supervision of manufacturing plant.

The former description presents an outlook of developed communications software for CORE, from the integrated information system point of view. Specific hardware and software modules are depicted in figure 1 for control of manufacturing cells, which communicate through its API's in CORE (PC). For Token Ring and SINEC L2 was developed PC software for its API's. However for ARTIC cards, besides PC software for its API's were developed specific tasks for the protocol control of connecting device. Elements of communication between the tasks of mentioned cards are outlined in figure 1. There is a specific control task for each port (task $\mathrm{x}$ ), and another one for communication (postman) from the PC and mentioned tasks through mailboxes. All developed software for CORE is written in $\mathrm{C} / 2$ language form IBM.

\section{CONTROL OF ROBOTS}

Control and monitoring of robots is made through a bi-directional communications link between communications server (CORE) and each robot, and its transfer of information is managed by an intermediate element implemented for a communications card (ARTIC), which importance is to be transparent to the whole system. Robot movings are controlled by a PC directly connected to it through an I/O card. The PC is under DOS and runs an only program which is a programming language interpreter called AML/2 (IBM, 1986). This is the language used for developing programs that will be executed by robots, given that has instructions for driving robot movings directly. The interpreter has a communications module able to execute the commands received through a serial communications port from the PC. With these commands (transactions) program can be selected for execution, starting, stopping, monitoring o modify its inner variables, etc., even any command defined in abstract form with independence of device.

AML/2 language has instructions that allow a robot program send information through a PC serial port. These informations are called events, that could be several manufacturing parameters such as: number of manufactured products, number of alarms, etc. Data that perform events and transactions are formatted in frames with headers that apply the link protocol (OSI level two), for the AML/2 communications module. A robot, in the same way that PLC's, is a cell in the manufacturing plant, and it has an only device name associated with it in the plant. The communications server sends commands to a robot in understandable format for the $\mathrm{AML} / 2$, issuing the name of destination device associated to the robot cell.

Each ARTIC card can communicate with 8 robots through the same RS-232 serial ports. These cards have a multitasking real-time operating system, that allows to execute a task for each communications port (IBM, 1995). All these tasks communicate with other called 
postman (Figure 1), through data structures named mailboxes with an only name. Each task has a mailbox with the same name that cell assigned to its serial port. Data received from CORE have a header with the name of destination cell. The postman puts data in the mailbox with same name. The task to which the mailbox is owned, takes the data, and puts them in a link level frame, and sends them to the port. When the robot replays to a transaction or send an event, the task reads the frame from the port, removes the link level headers and puts data to the mailbox owned to postman, adding a header with its own mailbox name. In this way the postman knows the cell where the message comes from. These data are sent to the PC for its further processing. The information system, organized in this way has the following advantages:

- Whole mobility of cells inside manufacturing plant. In flexible manufacturing plants, the cells are changed of place according the manufactured product. Connected port to specific robot could be changed with a little adjustment in a configuration file.

- Independence from the connected devices. A robot could be changed by any device controlled by a serial port, replacing the port associated task by other that applies the protocol of new device, without change in the software of CORE or the postman task.

- PC freedom of communications processing. ARTIC cards have memory devices that allow the storage of data until CORE can processing them. In this way data are not lost when the communications server is busy and a new frame is received.

\section{CONTROL OF PLC'S}

Used PLC's in several cells of manufacturing plant are SIMATIC S5-95U from SIEMENS, with an interface for SINEC L2. Communications between them is made through the field bus SINEC L2 (Siemens, 1994), also from SIEMENS, that applies the standard field bus called PROFIBUS (Bender, 1993) (DIN 19245 part 1 and 2 (Profibus, 1994)). This field bus is selected by its high speed of transference (1.5 Mbps maximum), and for to be a standard with products from many manufacturers. This will allow to add, in the future, directly to the bus, PLC's, actuators and sensors that apply the standard. The communications with PLC's is made in CORE through the card CP5412(A2) (Siemens, 1995), and the software for its correct performance (Siemens, 1995) (driver, firmware, library of functions, etc.).

CORE software has code to perform communications with CP5412 based in the four following functions supported by the driver: ihi open_dev for having driver access, ihi write for sending commands to the driver and information to other network nodes, ihi read for receiving the replays and ihi close for leaving the access to driver. There are two ways of using these functions. The first one is in synchronous mode, sending a request to the driver and waiting for the response. The second one is in asynchronous mode, without waiting a reply when the request is sent, and taking it by a late consult to driver. Software of CORE performs the calls to above mentioned functions by asynchronous mode.

The S5-95U PLC's allow three types of different L2 communications: standard link, AG-AG link and access to level 2 services. The standard link was selected for its simplicity, and for allowing the use of programs with included communications through SINEC L1 (a proprietary bus from Siemens), without modifications. This kind of communication allows the receiving 
and sending of telegrams type SDA and SDN to the SAP number 56, with a data length between 1 and 242 bytes. For implementing this kind of communications are coded in the PLC the location of sending and receiving mailboxes, and its coordination bytes.

Implemented commands for control of PLC's are: start, stop, initial conditions, write and read of date and hour. Events generated by PLC's and sent to CORE can be made for: start, stop, circuit beginning, circuit ending, alarm beginning and alarm ending. In addition to these events there is another that the PLC sends to CORE if a minute is spent without any type of communication. In this way is possible to know if there is a PLC with communication troubles. Events include the following informations. A number of sequence generated by the PLC for monitoring if an event is missed or not. The number of hundredth of seconds passed from last event, to know with precision, for instance, the time spent in he manufacturing of a circuit, the time elapsed in an alarm condition, etc. Present date and hour. Number of correct and faulty circuits to the moment. Event type referred by these informations. Error code related with this event.

\section{COMMUNICATIONS SERVER}

All communications are made using the $\mathrm{AS} / 400$ as mediator. For that have a great importance the three queues defined in it (Figure 1): cellinput, celloutput and cellevents. All queues are defined as FIFO type, and only celloutput has also a key. Any command from a monitoring node to a cell, should be sent to cellinput. When the cell replays, CORE will transfer the information to celloutput. Finally, monitoring node will read from this queue the reply, putting as searching key its own name. A key is necessary for celloutput because in it could have responses to different nodes with arbitrary order. Each command has a format that includes the following fields: origin node, destination cell, sequence number, command and parameters. Is in the command field where must be specified the task that should be performed by destination cell. These commands are defined in such a way that were independents of specific target devices.

All events generated in different cells will go to CORE, and this will retransmit them to cellevents for their processing by the AS/400. Queues from AS/400 have been used as mediators in communications by their ease and convenience of use. This is true as for developed software for CORE, in C/2 of IBM for DOS environment, like for developed software for monitoring nodes, in Visual Age from IBM for OS/2 WARP environment.

CORE performance is summarized following. It is in a endless loop where the different inputs of data are monitored alternately, such us, the keyboard, the cellinput queue, the ARTIC cards and the SINEC L2 card. When a command is read from cellinput queue or from keyboard, is tested if destination cell and feasible command are valid. If it is true, the command is stored in a table of pending transactions and is translated to an understandable format for destination cell. Depending on destination, CORE sends the translated command to the respective communications card (ARTIC0, ARTIC1 or SINEC L2). When an information is read, from ARTIC cards or SINEC L2 card, firstly a testing is made if it is a replay of a previous command, or an event. If it is a replay, is translated according to the device type, this information is added to the command previously stored in the pending transactions table and is 
sent to celloutput queue. If it is an event, is translated according to the device and output format and is sent to cellevents queue. This process is continuously made for attending quickly to communications cards and prevent the loss of data.

\section{RESULTS}

Current implementation of the integrated information system is under test and the main results are:

- Testing of CORE in the manufacturing plant (Marino and Poza, 1995) is being successful, and new events from operating robots and PLC's are added to event table for validation of manufacturing integrated information system.

- Traffic measurements on LAN performed by network analyzers (Marino, 1995) show that CORE usage of LAN presents a "utilization as percent of capacity" index of $1.06 \%$ for a 4 Mbps LAN, and $0.26 \%$ for $16 \mathrm{Mbps}$ LAN Therefore traffic impact from CORE in manufacturing integrated information system is negligible.

- Worst case measurements in data storage (Marino, 1995) reveals that CORE packets from its manufacturing area (16 SCARA robots and 30 PLC's), could spend less than 10 Gbyte/year, without filtering process from manufacturing integrated information system. Then low cost storage equipment by magnetic tapes or magneto-optical disks could be used.

- Flexibility in change of production tasks, and real-time control of each event in manufacturing plant through CORE, will reduce in several points the manufacturing costs, according to a recent forecast from the company staff.

\section{CONCLUSIONS}

Performances of CORE in the integrated information system are summarized as follows:

- This server is designed for attending communications with multiprotocol devices through standard communication interfaces like PROFIBUS and EIA-RS232C. In integrated information systems can be viewed as a multipurpose "black box" at area level, for controlling several manufacturing cells.

- Modularity in developed software allows to add or eliminate any similar above mentioned devices (PLC's from SIEMENS and IBM's Scara robots), without changes, until allowable maximum. Nevertheless in case of different devices, only should be modified or added the software modules that meet the functionality specified by its manufacturer.

- This implementation contributes to enhance communications transparency in flexible manufacturing plants with integrated information systems. Also its hardware structure based in a PC with coprocessor cards, simplifies communications equipment and reduces its cost. 


\section{REFERENCES}

Bender, K. (editor) (1993) Profibus: the field bus for industrial automation (ed. Prentice Hall). IBM (1986) AML/2 manufacturing control system, user's guide, PN67X1370.

IBM (1995) Real-time interface co-processor multiport/2, technical reference, software vols. 1,2 and 3.

IBM (1995) Real-time interface co-processor C language support version 1.02, user's guide.

IBM (1995) Real-time interface co-processor multiport/2, extended services, user's guide.

Marino, P. and Poza, F. (1995) Distributed system for assembly of SMT electronic equipment, 5th. National conference on computer aided project, planning and production. University of Minho, Guimarães (Portugal), May (30-31) and June (1).

Marino, P., Poza, F. and Nogueira, J.B. (1995) Distributed systems based on real-time communications cards. Mundo Electrónico (ed. Marcombo, Barcelona) 254 (Jan-Feb.), 3843.

Marino, P., Poza, F. and Nogueira, J.B. (1995) Multiport cards: performances and applications. Investigación y Ciencia (spanish edition of Scientific American), June, 74-75.

Marino, P. (1995) Enterprise communications: standards, networks and services (ed. RA-MA, Madrid).

Profibus (1994) Nutzerorganisation e. V.:PROFIBUS STANDARD, DIN 19245 Part 1 and 2.

Siemens (1994) SINEC: Industrial Communications Networks, Catalog Ik 10.

Siemens (1995) SINEC: Introduction to the CP5412(A2).

Siemens (1995) SINEC: FDL programming interface.

\section{BIOGRAPHY}

\section{PERFECTO MARINO}

Doctor on Telecommunications Engineering from the Polytechnic University of Madrid (Spain 1984). Professor of Electronic Technology Department (University of Vigo, Spain). Visiting scientist in the Computer Science Department of Carnegie Mellon University (Pittsburgh, USA 1988). Expert on Information Technology from the Commission of the European Union for the SPRINT (Luxembourg 1991) and COPERNICUS (Brussels, 1994) programs. Director of Digital Communications Division from the Applied Electronic Institute (University of Vigo, Spain). e-mail:pmarino(a),uvigo.es

\section{FRANCISCO POZA}

Electronic Engineer from the Industrial and Telecommunications Engineering University (Vigo, Spain 1986). Associate Professor in the Electronic Technology Department (University of Vigo, Spain). Researcher of Digital Communications Division from the Applied Electronic Institute (University of Vigo, Spain). e-mpaiła(a),uvigo.es

\section{JUAN B. NOGUEIRA}

Telecommunications Engineer from the Industrial and Telecommunications Engineering University (Vigo, Spain 1993). Associate Professor in the Electronic Technology Department (University of Vigo, Spain). Researcher of Digital Communications Division from the Applied Electronic Institute (University of Vigo, Spain). e-mail: nogueira(â)uvigo.es 